\title{
RapidScat Sigma-0 Measurements Validation
}

Ruaa Alsabah, Ali Al-Sabbagh, and Josko Zec

Department of Electrical and Computer Engineering

Florida institute of technology, Melbourne, FL, USA

\section{Introduction}

- RapidScat is a first Ku-band scatterometer that flew onboard the International Space Station (ISS) . ISS Sun-asynchronous orbit enables, for the first time, overlap with other satellite scatterometers that have been flying in Sun-synchronous orbits. RapidScat's primary objective had been global wind vector retrieval over sea surface from the measured normalized radar cross section $\left(\sigma^{0}\right)$

To assure measurement accuracy, careful scatterometer calibration and validation is required.

Cross-calibration among overlapping instruments is a common calibration method. This work explores the double-difference methodology to scatterometry.

- The methodology has been adopted for the cross-instrument calibration between RapidScat and Quikscat scatterometers simultaneously orbiting the Earth onboard two independent satellite platforms.

- Calculated biases may be used for measurement correction and reprocessing

- Table 1 compares the basic RapidScat and QuikScat configurations.

This work summarizes the comparison measurements data in the period from January 2015 to August 2016.

Table 1. RapidScat and QuikScat system comparison

\begin{tabular}{|c|c|c|}
\hline $\begin{array}{l}\text { Instrument } \\
\text { Frequency (Ku-band }\end{array}$ & $\begin{array}{l}\text { Seawinds (QS) } \\
36 \mathrm{GHz}\end{array}$ & $\frac{R^{2}}{136 \mathrm{pidscat}(\mathrm{RS})}$ \\
\hline $\begin{array}{l}\text { Prequency (ku-bandations } \\
\text { Polarations }\end{array}$ & $\frac{13.6 \mathrm{GHz}}{\text { VV-outer } \mathrm{HH} \text {-inner }}$ & $\frac{11.6 \mathrm{GHz}}{\mathrm{V} \text {-outer } \mathrm{HH} \text {-inner }}$ \\
\hline Daily coverage & $92 \%$ & $\begin{array}{l}\text { 65\% between } \\
\text { 58\% Th }\end{array}$ \\
\hline Mission \& Dates & $\begin{array}{l}\text { QuikSCAT } \\
6 / 1999-11 / 2009 \\
\text { (1) }\end{array}$ & Space Station \\
\hline Orbit type & Sun-synchronous & vachronous \\
\hline Resolution $\left(0^{\circ}\right)$ & $\begin{array}{l}\text { Egg: } 25 \times 363 \mathrm{~km} \\
\text { cis: }\end{array}$ & Egg: $26 \times 37 \mathrm{~km}$ \\
\hline Swath $(\mathrm{km})$ & 1400,18 & 900,1100 \\
\hline 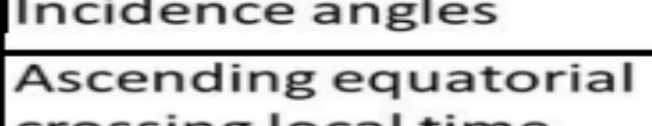 & & \\
\hline $\begin{array}{l}\text { crossing local time } \\
\text { Altitude (nominal) }\end{array}$ & & $375-435$ \\
\hline Period & & \\
\hline
\end{tabular}

\section{Data Sets}

RapidScat data have been extracted from the Level 2A (L2A). And from level 1C (L1C) for QuikScat data product.

- The data products were generated by the Physical Oceanography Distributed Active Archive Center at JPL.

- Level 2A data products come in three versions, 1.1, 1.2, and 1.3, with 1.2 replacing 1.1 after $08 / 15 / 2015$; version 1.3 is intended as a replacement and continuation of the Version 1.1, and Version 1.2 data forwarded from 02/11/2016. - level 1C (L1C) represent the geo-located and averaged $\sigma^{0}$ measurements and wind retrievals during the non-spinning mode

\section{Calibration Method}

The steps for the RapidScat/QuikScat cross-calibration process are:

- Grid $\sigma^{0}$ data into $1^{\circ} \quad \mathrm{x} 1^{\circ}$ resolution pixels over the globe.

- Apply Conservative filters.

- The same $1^{\circ} \times 1^{\circ} \quad \sigma^{0}$ grid is populated with the environmental data: wind speed (WS) and wind direction (WD).

- The modeled $\sigma^{0}$ s are calculated in each $1^{\circ} \times 1^{\circ}$ pixel for both instruments from Geophysical Model Function.

- GMF of NSCAT-2014 was used in this research to calculate $\sigma^{0}$ for horizontal and vertical polarizations covering a full range of relative wind directions $\left(0-180^{\circ}\right)$, wind speeds $(0-70$ $\mathrm{m} \cdot \mathrm{s}-1)$ in steps of $0.2 \mathrm{~m} \cdot \mathrm{s}-1$, and incidence angles between $16^{\circ}$ and $66^{\circ}$ with a $0.5^{\circ}$ resolution. Figure 1 shows the RapidScat corresponding readings to NSCAT-2014 GMF.

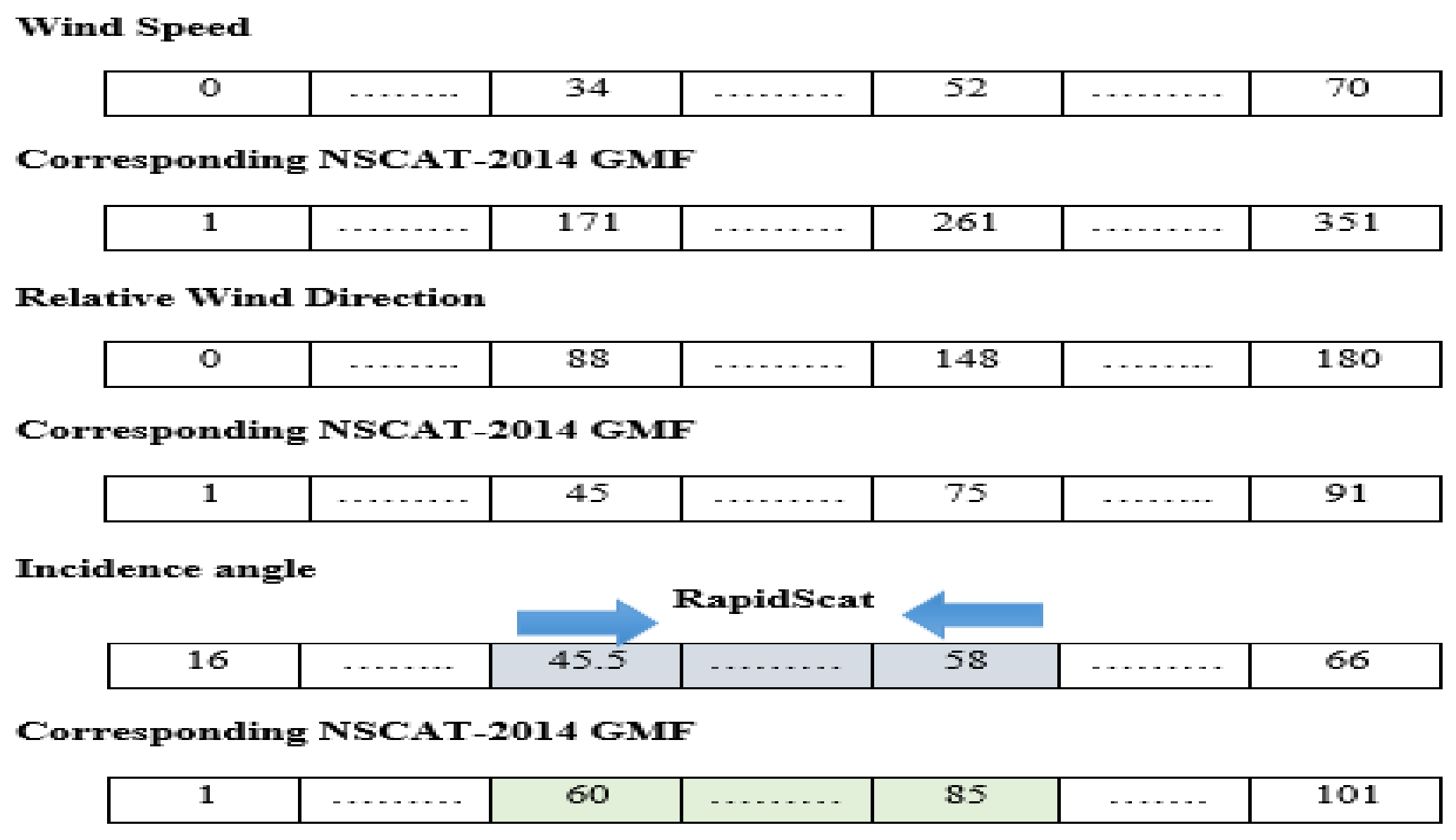

Fig. 1 Corresponding readings to NSCAT-2014 GMF.

- The modelled modeled $\sigma_{\mathrm{GMF}}^{0}$ is calculated from the GMF at given incidence angles $\theta$, wind speed $w s$, wind direction relative to scatterometer antenna azimuth $\chi$ and polarization $\mathrm{p}$ :

$$
\begin{aligned}
& \sigma_{G M F, R S}^{0}\left(\theta_{R S}, w s, \chi_{R S}, p_{R S}\right) \\
& \sigma_{G M F, Q S}^{0}\left(\theta_{Q S}, w s, \chi_{Q S}, p_{Q S}\right)
\end{aligned}
$$

- To build the single-difference (SD) bias, the modeled $\sigma_{\mathrm{GMF}}^{0}$ can be compared with the observed $\sigma_{\mathrm{RS}}^{0} / \sigma_{\mathrm{QS}}^{0}$. This bias between measured and modelled (expected) $\sigma^{0}$ is calculated as:

$$
\begin{aligned}
& S D_{-} R S=\sigma_{R S}^{\circ}-\sigma_{G M F, R S}^{\circ}(\theta, W, X, p) \\
& S D_{-} Q S=\sigma_{Q S}^{\circ}-\sigma_{G M F, Q S}^{\circ}(\theta, W, X, p)
\end{aligned}
$$

- Single differences calculated previously can be subtracted to estimate doubledifference and thus enable relative calibration between RapidScat and QuikScat completing the cross-calibration process.

$D D(\theta, W, X, p)=S D_{-} R S(\theta, W, X, p)-S D_{-} Q S(\theta, W, X, p)$

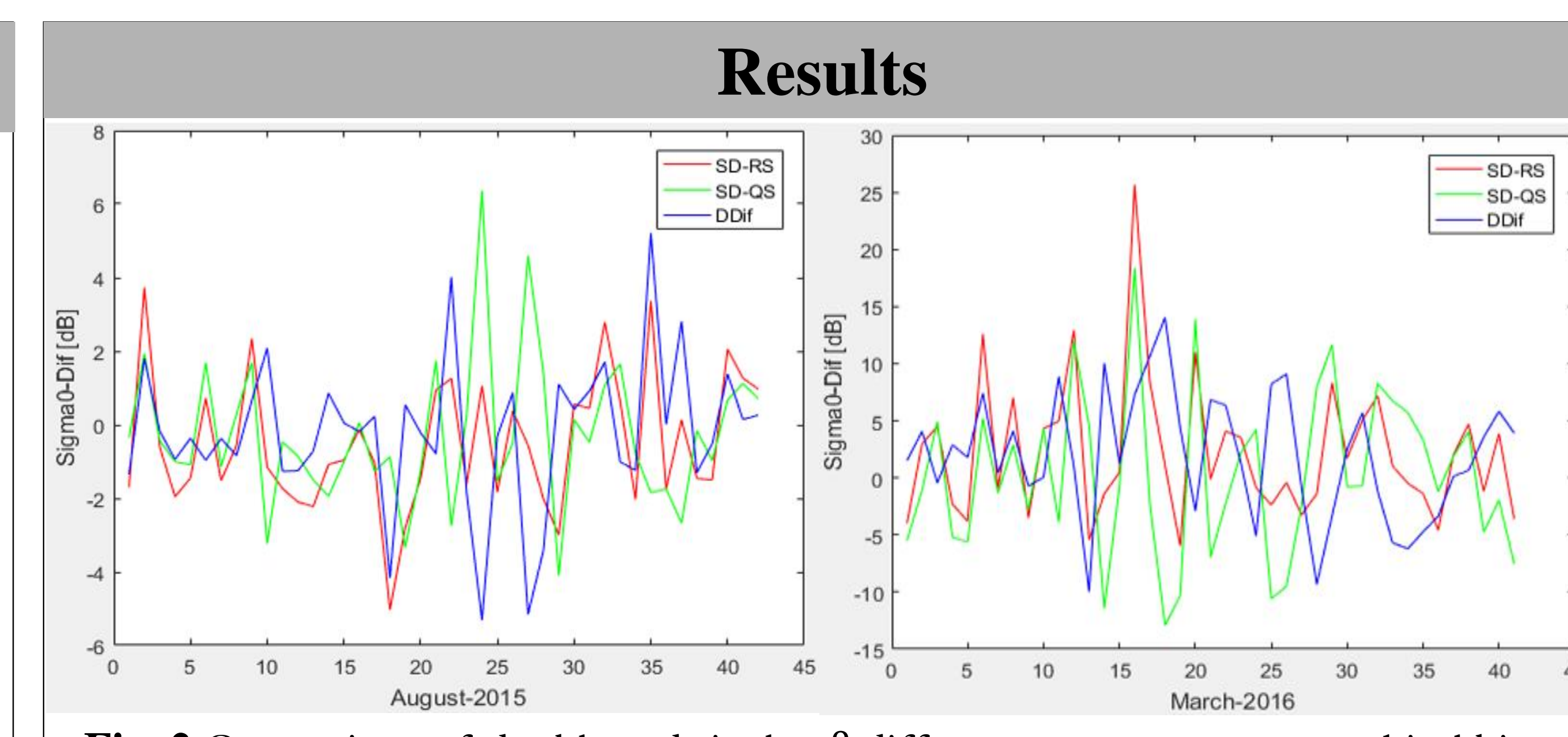

Fig. 2 Comparison of double and single $\sigma^{0}$ differences per some geographical bin August 2015 (left) March 2016 (right)

- Figure 2 present an example of a random selection of bins was chosen to show fluctuations of RapidScat and QuikScat single differences and the double difference per geographical bin. Biases are generally centered around 0dB mean $\sigma^{\mathrm{o}}$ difference, but exhibit large outliers in sporadic individual bins.

- Moreover the entire period was examined to assess the monthly averaged variations of double differences within $0.5 \mathrm{~dB}$ at the beginning of the comparison period, until approaching the maximum double difference bias of 2 dB in January 2016 as shown in figure 3 .

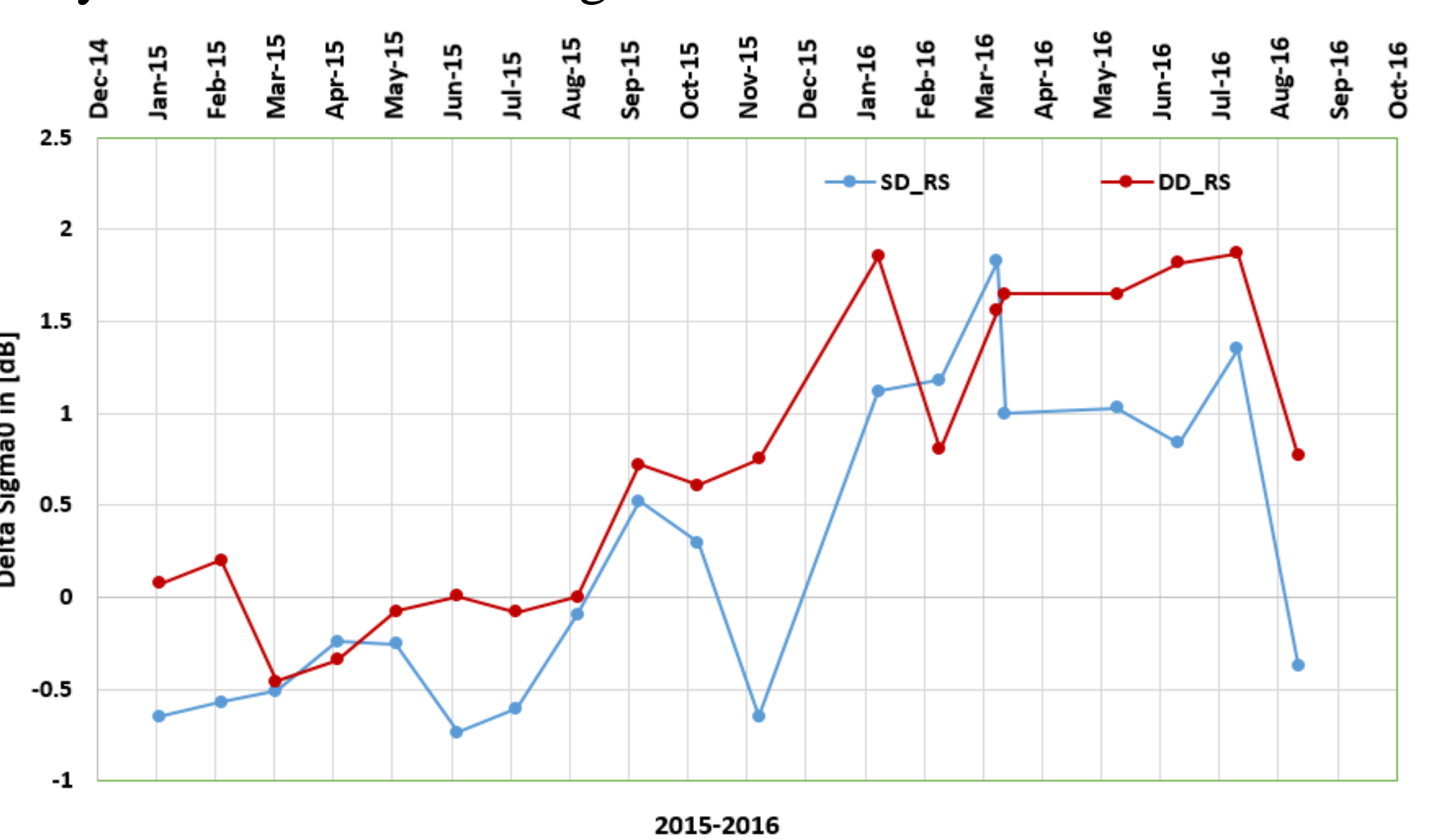

Fig. 3 Monthly average RapidScat single-difference and double-difference

\section{Conclusion}

- Results obtained over 2 years observation period indicate consistency between two instruments in the first year, until the increased $\sigma^{\circ}$ divergence coinciding with detection of low SNR periods in RapidScat data 